\title{
Medial blepharosynechioplasty: a new surgical concept for severe dry eye
}

This article was published in the following Dove Press journal:

Clinical Ophthalmology

21 June 2012

Number of times this article has been viewed

\author{
Tsugihisa Sasaki ${ }^{1,2}$ \\ Taeko Ota ${ }^{3}$ \\ Youko Ookura ${ }^{4}$ \\ Kazuhisa Sugiyama' \\ 'Department of Ophthalmology, \\ Kanazawa University School of \\ Medicine, Kanazawa, Ishikawa; \\ ${ }^{2}$ Department of Ophthalmology, \\ Fukui Prefectural Hospital, Fukui; \\ ${ }^{3}$ Department of Ophthalmology, \\ Tonami General Hospital, Tonami-city, \\ Toyama; ${ }^{4}$ Department of \\ Ophthamology, Saiseikai Kanazawa \\ Hospital, Kanazawa, Ishikawa, Japan
}

Correspondence: Tsugihisa Sasaki Sasaki Eye Clinic, 5-2-6 Mikuni-higashi, Mikuni, Sakai, 913-0016, Japan Tel +81776880033

Fax +81776880233

Email sasatsug@gmail.com
Background: The purpose of this work was to report on the performance of medial blepharosynechioplasty (MBSP), a newly devised technique for treating severe dry eye.

Methods: In this retrospective, nonrandomized clinical trial, three cases with severe dry eye (Sjögren's syndrome) associated with repeated punctal plug loss were treated using MBSP to create a synechia between the upper and lower lid medial borders of the puncta to suppress the lacrimal pump.

Results: Postoperative follow-up showed improvement in the corneal condition in all three cases that persisted for 12-35 months. None of the patients had visual impairment.

Conclusion: MBSP is a promising treatment for severe dry eye and merits further study.

Keywords: dry eye, lacrimal pump suppression, medial blepharosynechioplasty

\section{Introduction}

Although punctal plug and occlusion of the lacrimal puncta is an established treatment for dry eye, ${ }^{1-3}$ the risk of complications, including epiphora, intrusion or extrusion of punctal plugs, canalicular stenosis, ${ }^{1,4}$ and inadequate results in cases of monopunctal closure, have frustrated surgeons when performing surgery involving permanent closure of the puncta. Development of alternative surgical methods to suppress the lacrimal pump and tear evaporation with fewer complications would represent a valuable advance in producing a milder effect than upper and lower punctal plugging.

We encountered three patients after dacryocystorhinostomy, who developed blepharosynechia bordering the punctum characterized by epiphora, although the lacrimal ducts remained patent. Epiphora improved after excision of their blepharosynechia. These observations suggested that blepharosynechia-induced epiphora was caused by suppression of the lacrimal pump, which in turn led to us devising medial blepharosynechioplasty (MBSP) as a novel treatment for dry eye.

\section{Methods}

The study protocol and data collection methods were approved by the institutional review board of Fukui Prefectural Hospital. This study reports on three patients suffering from repeated punctal plug loss.

\section{Surgical procedure}

The infratrochlear nerve was anesthetized using an injection of a mixture of $0.01 \%$ epinephrine and 2\% lidocaine. Two strips of tissue from the palpebral conjunctiva, 
each measuring $2 \times 3 \times 0.5 \mathrm{~mm}$, were excised from the medial side of the upper and lower puncta to facilitate formation of a synechia (Figure 1). Eyelid tissues in the immediate vicinity of the puncta were sewn together with 7-0 nylon in conjunction with insertion of a lacrimal probe into the canaliculus to
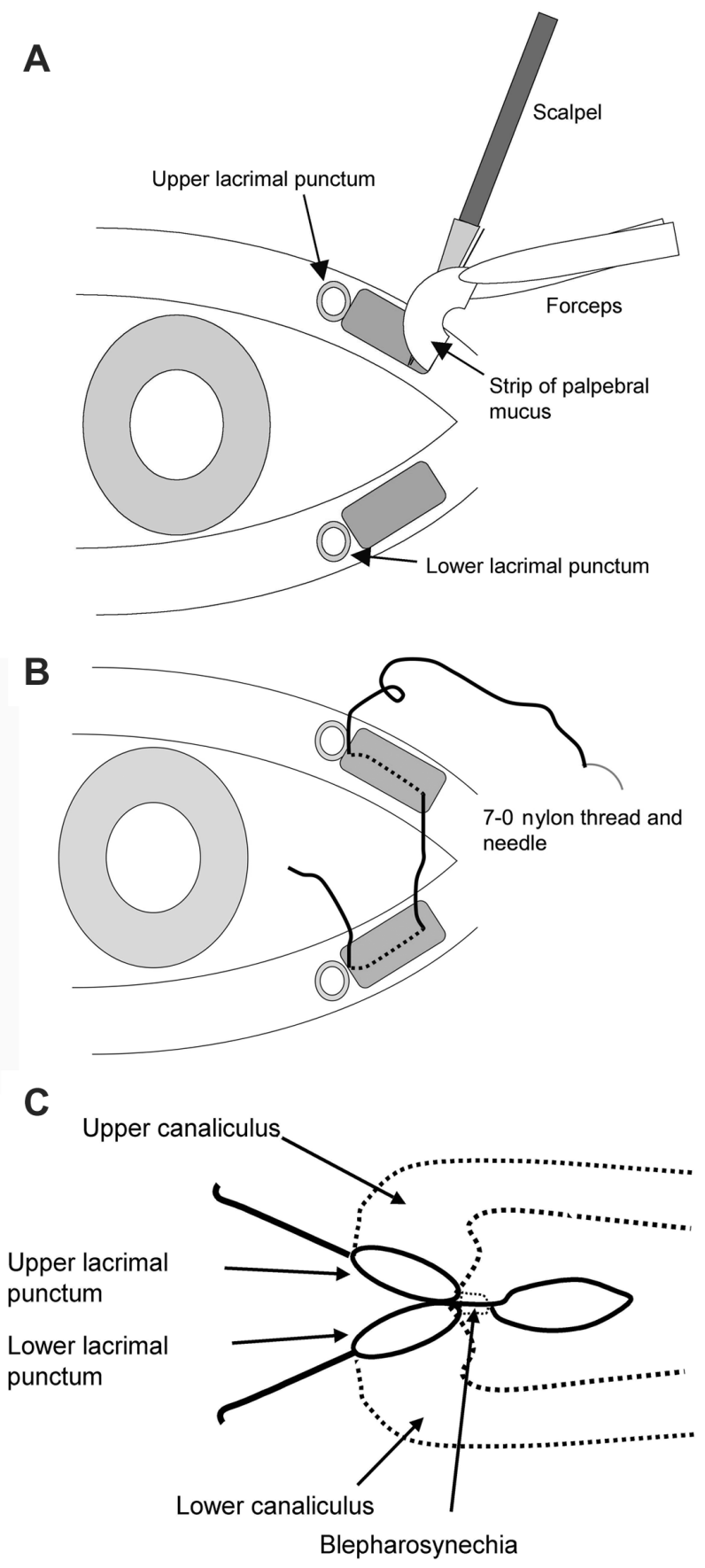

Figure I Schematic view of medial blepharosynechioplasty (MBSP) in the right eye. (A) Excision of two strips of palpebral conjunctiva, each measuring $2 \times 3 \times 0.5 \mathrm{~mm}$ (width $\times$ length $\times$ thickness), from the medial side of the upper and lower puncta to facilitate formation of a synechia. (B) The incised eyelids were sewn together in the immediate vicinity of the puncta with 7-0 nylon in conjunction with insertion of a lacrimal probe into the canaliculus to prevent damage to it. (C) Enlarged view near the lacrimal puncta after MBSP. prevent damage to it. Antibiotic ophthalmic drops and $0.1 \%$ fluorometholone were applied postoperatively for 2-4 weeks. The area of the uncovered ocular surface was calculated using Navis (Nidek, Gamagori, Japan) and Photoshop CS (Adobe Systems, San Jose, CA) software from slit-lamp images.

\section{Patient I}

Patient 1 was a 73-year-old woman who had a 20-year history of Sjögren's syndrome and keratoconjunctivitis sicca. Schirmer's test was $0 \mathrm{~mm}$ in each eye preoperatively. Slit-lamp examination revealed a bilateral shortened tear film break-up time ( 2 seconds, $\mathrm{R}=\mathrm{L}$ ) and moderate superficial punctate keratoconjunctivitis. She underwent MBSP in her left eye. A postoperative Schirmer's test was still $0 \mathrm{~mm}$ in both eyes. Slit lamp examination showed improvement of superficial punctate keratoconjunctivitis in the left eye and an increase in tear meniscus height from $0.1 \mathrm{~mm}$ to $0.2 \mathrm{~mm}$ (Figure 2). The area of the uncovered ocular surface was reduced $78.3 \%$ postoperatively from the preoperative value. Postoperative follow-up for 35 months showed no recurrence of severe superficial punctate keratoconjunctivitis.

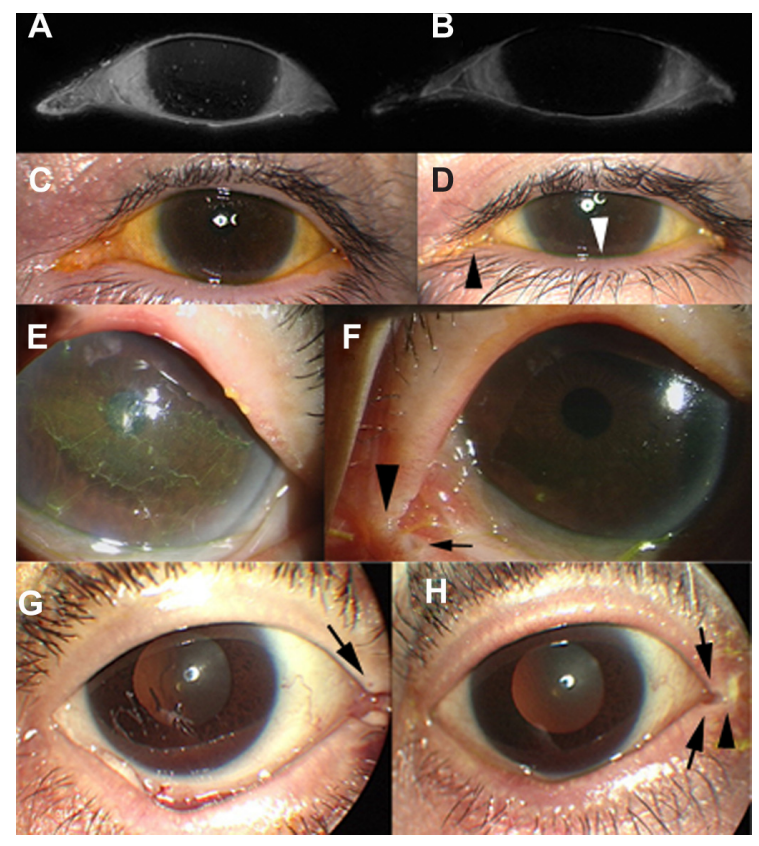

Figure 2 Preoperative and postoperative photographs of patients I-3. (A) Preoperative photograph of fluorescent dye-stained ocular surface in patient I. White spots imply superficial punctate keratitis. (B) Postoperative photograph of fluorescent dye-stained ocular surface in patient I. Superficial punctate keratitis improved. (C) Preoperative photograph of same patient as in Figure 2A. (D) Postoperative photograph of same patient as in Figure 2B. Tear meniscus height elevation (white arrow head) is identified in patient I. Synechia near the puncta are identified (arrow). (E) Preoperative photograph of patient 2. Severe dry eye is observed. (F) In patient 2, improvements in corneal condition and blepharosynechia (black arrow head) are observed following MBSP. Puncta (arrow) near synechia are still open. (G) Preoperative photograph of patient 3. Corneal filamentosa is observed. $(\mathbf{H})$ In patient 3, improvements in corneal filamentosa and blepharosynechia (black arrow head) are observed postoperatively. Puncta (arrow) near synechia are still open. 


\section{Patient 2}

Patient 2 was a 79-year-old woman who had a long history of Sjögren's syndrome, dry eye, and bilateral persistent filamentary keratopathy (Figure 2). Schirmer's test was $0 \mathrm{~mm}$ in both eyes. She underwent MBSP in her left eye. Filamentary keratopathy improved dramatically after the operation (Figure 2). These improvements persisted postoperatively for 30 months.

\section{Patient 3}

Patient 3 was a 76-year-old woman who had a long history of Sjögren's syndrome and chronic dacryocystitis on the left side. Dacryocystitis was treated with dacryocystorhinostomy. She subsequently showed severe superficial punctate keratoconjunctivitis in each eye, at which time Schirmer's test was $1 \mathrm{~mm}$ in each eye. She underwent MBSP in her right eye one year after her dacryocystorhinostomy (Figure 2). Her superficial punctate keratoconjunctivitis improved. The postoperative Schirmer's test was $5 \mathrm{~mm}$ in the right eye and $2 \mathrm{~mm}$ in the left eye. Improvement in filamentary keratopathy was observed at her 12-month postoperative follow-up visit.

\section{Discussion}

MBSP increased the quantity of tear fluid in the treated eyes. Punctal plug, punctal closure, and punctal tarsorrhaphy are surgical treatments for severe dry eye. ${ }^{3}$ MBSP resembles punctal tarsorrhaphy, but there is a difference in the patency of the lacrimal passage in MBSP. Conventional punctal tarsorrhaphy was devised for complete blockage of lacrimal drainage. However, MBSP aims to reverse the pump mechanism partially. The mechanism of action for MBSP is obscure. We hypothesize that MBSP blocks movement of the puncta and canaliculi during blinking and reduces evaporation of tears from the ocular surface through the minitarsorrhaphy. However, reducing evaporation is not thought to cause a major effect because MBSP improves the condition of the cornea, even in the uncovered area of the cornea. Moreover, tarsorrhaphy-induced ocular coverage reduced tear evaporation by $22 \%$ in patient 1 . It has also been postulated that apposition of the puncta during blinking is an important component of the valve mechanism in the lacrimal pump. ${ }^{5}$ By creating misalignment of the puncta during blinking, MBSP may interfere with this valve mechanism.

Periorbital injection of botulinum toxin has been reported to improve dry eye $\mathrm{e}^{6,7}$ by mechanisms involving paralysis of the orbicularis oculi muscle around the canaliculi. MBSP may operate by a mechanism similar to that of botulinum toxin injection. Treatments involving pump suppression may well become a treatment of choice in the future. In all cases, the lacrimal ducts remained patent with no visual impairment and no punctal displacement. Further studies, including a clinical trial of MBSP utilizing a control group, are necessary.

\section{Disclosure}

The authors did not receive financial or material support from the manufacturer of any commercial product discussed in this paper.

\section{References}

1. Willis RM, Folberg R, Krachmer JH, Holland EJ. The treatment of aqueous-deficient dry eye with removable punctal plugs. A clinical and impression - cytologic study. Ophthalmology. 1987;94(5):514-518.

2. Kaido M, Goto E, Dogru M, Tsubota K. Punctal occlusion in the management of chronic Stevens-Johnson syndrome. Ophthalmology. 2004; 111(5):895-900.

3. Murube J, Murube E. Treatment of dry eye by blocking the lacrimal canaliculi. Surv Ophthalmol. 1996;40(6):463-480.

4. Balaram M, Schaumberg DA, Dana MR. Efficacy and tolerability outcomes after punctal occlusion with silicone plugs in dry eye syndrome. Am J Ophthalmol. 2001;131(1):30-36.

5. Doane MG. Blinking and the mechanics of the lacrimal drainage system. Ophthalmology. 1981;88(8):844-851.

6. Spiera H, Asbell PA, Simpson DM. Botulinum toxin increases tearing in patients with Sjögren's syndrome: a preliminary report. J Rheumatol. 1997; 24(9):1842-1843.

7. Sahlin S, Chen E, Kaugesaar T, Almqvist H, Kjellberg K, Lennerstrand G. Effect of eyelid botulinum toxin injection on lacrimal drainage. Am J Ophthalmol. 2000;129(4):481-486.
Clinical Ophthalmology

\section{Publish your work in this journal}

Clinical Ophthalmology is an international, peer-reviewed journal covering all subspecialties within ophthalmology. Key topics include: Optometry; Visual science; Pharmacology and drug therapy in eye diseases; Basic Sciences; Primary and Secondary eye care; Patient Safety and Quality of Care Improvements. This journal is indexed on

\section{Dovepress}

PubMed Central and CAS, and is the official journal of The Society of Clinical Ophthalmology (SCO). The manuscript management system is completely online and includes a very quick and fair peer-review system, which is all easy to use. Visit http://www.dovepress.com/ testimonials.php to read real quotes from published authors. 